\section{Expanded Chemoprophylaxis Offered in Response to a Case of Meningococcal Meningitis in an Elementary School — Indiana, 2015}

Deborah A. McMahan, MD ${ }^{1}$; Erika D. Pitcher, MPH ${ }^{1}$;

Mindy R. Waldron ${ }^{1}$; Amanda S. Billman, $\mathrm{MPH}^{2}$; Shawn M. Richards ${ }^{2}$; Pamela R. Pontones, $\mathrm{MA}^{2}$; Joan M. Duwve, $\mathrm{MD}^{2}$

On December 11, 2015, the Fort Wayne-Allen County (Indiana) Department of Health was notified by a local hospital laboratory of a suspected case of meningococcal meningitis based on Gram stain results of cerebrospinal fluid. The county health department interviewed close family members and friends of the patient to establish an infectious period, timeline of events, and possible exposures. Close medical and household contacts were offered chemoprophylaxis (1). This case was associated with an elementary school. The patient had intermittent, close, potentially face-to-face contact with many students, and was reported to have had a persistent, productive cough throughout the exposure period. In light of these unusual circumstances, and the fact that elementary school-aged children are not routinely vaccinated against meningococcal disease, ${ }^{*}$ local and state health officials, with CDC support, decided to offer chemoprophylaxis to the patient's contacts. A total of 581 child and adult contacts were identified.

Local, state, and federal public and private health care providers partnered in planning and implementing the distribution of prophylaxis and developing a communication strategy to inform parents and the community. This public-private partnership resulted in a high level of compliance with public health recommendations and minimal disruption to families and the elementary school.

The chemoprophylaxis clinic took place at the school on December 15, 2015. After discussion with CDC, ciprofloxacin oral suspension was offered according to published guidelines as the preferred antibiotic because of its ease of administration as a single dose, the need for varying dosages because of the number of children weighing $<55$ pounds $(<25 \mathrm{~kg}$ ), and the concern that some children might not be able to swallow pills (1). Parkview Regional Medical Center, a local nonprofit hospital, provided pharmacy services to facilitate and distribute appropriate dosages of ciprofloxacin $(20 \mathrm{mg} / \mathrm{kg}$, orally, up to a maximum of $500 \mathrm{mg})$ (1) and to answer questions regarding potential drug interactions, indications, and safety. Ceftriaxone was offered to pregnant or lactating women, and ceftriaxone or rifampin was offered to persons with contraindications to or precautions for ciprofloxacin use (2).

\footnotetext{
*http://www.cdc.gov/vaccines/schedules/index.html.
}

Among 581 persons identified as contacts, 496 (85\%) received chemoprophylaxis; among these persons, 449 (91\%) received chemoprophylaxis at the clinic, including 335 students and 114 school faculty members or volunteers. In addition, 12 health care workers, eight close household contacts, and 27 peripheral event contacts received chemoprophylaxis outside of the clinic. The county health department offered home delivery of medication for persons who were unable to attend the clinic, and the hospital pharmacy also stored the medication at the hospital to facilitate pickup. Despite these efforts, $15 \%$ of contacts chose not to pick up the medication for undisclosed reasons. No immediate adverse events were reported from those who received ciprofloxacin or rifampin provided at the clinic.

On December 21, 2015, the patient was confirmed to have Neisseria meningitidis serogroup B infection by testing performed at CDC. The patient fully recovered, and no additional cases were identified.

Although chemoprophylaxis of persons other than close contacts is not routinely recommended in response to a single case of meningococcal meningitis, in unique circumstances when expanded meningococcal chemoprophylaxis is warranted, it is important to identify a well-defined target group and ensure that all persons within the target group receive antibiotics within a short time frame. In this case, the communication framework and professional relationships developed during the 2009 H1N1 influenza pandemic created a multidisciplinary infrastructure that facilitated the investigation and response. Providing the appropriate chemoprophylaxis for approximately 500 persons in 2 days with minimal school disruption is an indicator of the strength of the local health partnerships with schools, hospitals, and health care providers, and of the lessons learned from previous public health emergencies.

\section{Acknowledgments}

Sarah A. Meyer, MD; Stacey W. Martin, MSc; Jessica R. MacNeil, $\mathrm{MPH}, \mathrm{CDC}$.

\footnotetext{
${ }^{1}$ Fort Wayne-Allen County Department of Health, Indiana; ${ }^{2}$ Epidemiology Resource Center, Indiana State Department of Health.

Corresponding author: Erika Pitcher, erika.pitcher@co.allen.in.us, 260-449-4838.
}

\section{References}

1. American Academy of Pediatrics. Meningococcal infections. In: Kimberlin DW, Brady MT, Jackson MA, Long SS, eds. Red book: 2015 report of the Committee on Infectious Diseases. 30th ed. Elk Grove Village, IL: American Academy of Pediatrics; 2015:547-58.

2. Meningitis CA. In: Heymann D, ed. Control of communicable diseases manual. 20th ed. Washington, DC: APHA Press; 2014:404-13. 